\title{
The Spectra of Bright Near-IR Clusters in M82
}

\author{
A. Lançon ${ }^{(1)}$, M. Mouhcine ${ }^{(1)}$, E. Chané(1), L.J.Smith ${ }^{(2)}$, \\ J.S. Gallagher ${ }^{(3)}$, N. Förster Schreiber ${ }^{(4)}$, W. Vacca ${ }^{(5)}$, R. de Grijs ${ }^{(6)}$, \\ R.W. O'Connell (7) \\ (1) Observatoire de Strasbourg (UMR 7550), France; (2) University \\ College London, UK; (3) University of Maddison, Wisconsin, USA; (4) \\ Leiden University, The Netherlands; (5) NASA Ames Research Centre, \\ USA; (6) Sheffield University, UK; (7) University of Virginia, USA
}

\begin{abstract}
Based on new spectra spanning wavelengths from 0.8 to $2.4 \mu \mathrm{m}$, we study the properties of bright near-IR clusters in M82. We focus on age and extinction, which are critical parameters when one uses dynamical masses to constrain the stellar IMF. The modelling of red supergiant evolution by various authors leads to very significant differences in synthetic cluster spectra. NearIR fluxes alone therefore do not rule out a normal IMF for cluster F, previously found to be deficient in low mass stars. Combined optical and near-IR studies are being undertaken.
\end{abstract}

The spectra of four bright near-IR clusters and of the nucleus of M82 were obtained with SpeX on IRTF (NASA/Hawaii). Synthetic spectra of cluster populations were constructed using the evolutionary tracks of the Padova group, a standard IMF, and the BaSeL library of stellar spectra, into which new SpeX supergiant spectra were inserted. Note that at solar metallicity the $T_{\text {eff }}$ distribution of the red supergiants populating the Padova isochrones peaks around $3900 \mathrm{~K}$ ( $\mathrm{M}$ stars contribute relatively little), while the predominant $\mathrm{T}_{\text {eff }}$ is cooler with the tracks of the Geneva group (at least for a range of ages). Tracks including rotation are different again.

We built $\chi^{2}$-maps in age-extinction space for all targets and find that: (1) all targets are dominated by red supergiants, including cluster F and the previously overlooked cluster observed in the Eastern "fossil starburst" region; (2) with the Padova tracks, age discrimination is strong between 5 and $10 \mathrm{Myr}$, but weak between 12 and $60 \mathrm{Myr}$; for cluster F, our best fit is at $10 \mathrm{Myr}$ with $\mathrm{A}_{V}=1.9$, which would make the dynamical mass of Smith \& Gallagher (2001, MNRAS 326, 1027) consistent with a normal IMF; their age of $\sim 60$ Myr is however not excluded;

(3) estimated ages depend strongly on the assumed metallicity and stellar rotation; work with a variety of tracks is necessary.

More robust constraints on age, intrinsic luminosity and thus the IMF will be obtained by using optical and near-IR spectra jointly. This will also constrain red supergiant evolution models. 